\title{
Some remarks on "State Estimation and Fault Diagnosis of Labeled Time Petri Net Systems with Unobservable Transitions"
}

\author{
Zhou He, Zhiwu Li, Alessandro Giua, Francesco Basile, and Carla Seatzu,
}

\begin{abstract}
In this paper we comment on the algorithm proposed in the paper mentioned in the title to define and construct a graph, called Modified State Class Graph (MSCG), which summarizes all possible evolutions of a Time Petri net. We first show that under the assumptions mentioned in such a paper, the proposed graph could be infinite. Then, we underline the requirement of revising the notation and adding some information on certain edges of the graph. Finally, we remark that the current version of the algorithm does not consider all possible evolutions of the net system. In the final part of the manuscript we propose a revised algorithm for the definition and construction of the MSCG that overcomes all such limitations.
\end{abstract}

Published as:

Zhou He, Zhiwu Li, Alessandro Giua, Francesco Basile, and Carla Seatzu. "Some remarks on "State Estimation and Fault Diagnosis of Labeled Time Petri Net Systems with Unobservable Transitions"," IEEE Trans. on Automatic Control, Vol. 64, No. 12, pp. 5253-5259, 2019. DOI: 10.1109/TAC.2019.2910168.

Z. He is with the College of Mechanical and Electrical Engineering, Shaanxi University of Science and Technology, Xi'an 710021, China (email: hezhou@sust.edu.cn).

Z. W. Li is with the Institute of Systems Engineering, Macau University of Science and Technology, Taipa, Macau and also with School of Electro-Mechanical Engineering, Xidian University, Xi'an 710071, China (email: zhwli@xidian.edu.cn).

A. Giua is with the DIEE, University of Cagliari, Cagliari 09124, Italy (email: giua@unica.it).

F. Basile is with the Dipartimento di Ingegneria dell'Informazione, Ingegneria Elettrica e Matematica applicata, Università di Salerno, Italy (email: fbasile@unisa.it).

C. Seatzu is with the DIEE, University of Cagliari, Cagliari 09124, Italy (email: seatzu@unica.it). 


\section{INTRODUCTION}

In a recent paper Basile et al. [1] introduced a graph called Modified State Class Graph (MSCG) to describe in a compact and exhaustive manner all possible evolutions of a labeled Time Petri net system. The definition of the MSCG was given in an algoritmic way, namely it was based on the algorithm used for its construction. In this paper we show that the algorithm presented in [1] to define and construct the MSCG is not correct. In particular:

- The assumptions in [1] do not ensure the finiteness of the MSCG, as claimed. We show this via a counterexample.

- The notation used to define the time spent by the net system in a given class (node) of the graph may affect the correctness of the way some constraints, used to describe the possible evolutions on the net system, are written.

- Finally, the algorithm in [1] does not guarantee, as claimed, that all possible evolutions of the net system are described in the MSCG.

In this Technical Note we provide a revised version of the algorithm in [1] that overcomes the above limitations. We also prove that, under the assumptions that the TPN is bounded and there exists no repetitive sequence that may fire in zero time, the MSCG constructed using the revised algorithm is finite, which is equivalent to prove that the algorithm terminates in a finite number of steps.

We conclude this section with three important remarks. First, the example proposed in [1] is correct since it does not violate the missing assumption, the problem in the notation does not appear there, and the MSCG actually provides all possible evolutions of the net system. Second, in [1] a procedure for the state estimation and fault diagnosis of labeled Time Petri nets is proposed based on the MSCG. The correctness of such results is not affected by the modifications introduced in the algorithm defining the MSCG. Indeed, the resulting MSCG mantains the structure and the features that were assumed when such results have been derived. Finally, the MSCG has been recently used in [2] to perform diagnosability analysis. Again, the example in [2] is correct and the results proposed there still apply when using the revised algorithm to construct the MSCG.

\section{The Modified State Class Graph}

A Time Petri net (TPN) is defined as a pair $N_{d}=(N, Q)$ where $N=(P, T$, Pre, Post $)$ defines the net structure and $Q: T \rightarrow \mathbb{R}_{0}^{+} \times\left(\mathbb{R}_{0}^{+} \cup\{\infty\}\right)$ defines the set of static intervals associated with transitions [1]. In particular, $P$ is the set of places, $T$ is the set of transitions, and Pre $: P \rightarrow \mathbb{N}$ and Post $: P \rightarrow \mathbb{N}$ are the preand post-incidence functions that specify the arcs [3]. In the following, the static lower bound of transition $t_{i}$ is denoted $l_{i}$, while its static upper bound is denoted as $u_{i}$.

A TPN $N_{d}$ with a marking $M_{0}$ at the initial time instant $\tau_{0}=0$ is called a marked TPN, or a TPN system, and is denoted as $\left\langle N_{d}, M_{0}\right\rangle$.

In this paper, as in [1], we assume that a labeling function $\overline{\mathcal{L}}: T \rightarrow L \cup\{\varepsilon\}$ assigns to each transition $t \in T$ either a symbol from a given alphabet $L$ or the empty string $\varepsilon$. The label of a transition represents the output 
it produces when firing.

The Modified State Class Graph (MSCG) is a directed graph whose nodes are called classes. With each class is associated a state of the net, namely a reachable marking $M$ and a set of inequalities $\Theta$ that define the timing constraints pertaining to all transitions enabled at $M$. Such inequalities depend on a certain number of variables, denoted $\Delta$ variables, which take into account how much time a transition has been enabled. The way such constraints are defined, as well as the way the $\Delta$ variables are initialized and updated, is clarified by Algorithms 1 and 2 in Section V. Edges are labeled as $\left(t, \gamma, \Delta \in\left[l^{*}, u^{*}\right]\right)$, where $t$ is the transition whose firing leads from the marking in the tail node to the marking in the head node; $\gamma$ is the label associated with $t$, and $\Delta \in\left[l^{*}, u^{*}\right]$ is a constraint on the time spent in the tail node of the edge. The upper bounds $l^{*}$ and $u^{*}$ are functions of $\Delta$ variables in the path from the root node to the head node of the edge at hand. As discussed in Section V where Algorithms 1 and 2 are presented, a fourth entry is added on certain edges to link $\Delta$ variables from a class to the next one when this is needed.

The goal of the MSCG relative to a TPN system $\left\langle N_{d}, M_{0}\right\rangle$ is that of providing a compact and exhaustive representation of all its possible evolutions. In more detail, a time-transition sequence $\left(t_{i_{1}}, \tau_{1}\right)\left(t_{i_{2}}, \tau_{2}\right) \ldots\left(t_{i_{k}}, \tau_{k}\right) \in$ $\left(T \times \mathbb{R}_{0}^{+}\right)^{*}$ is firable at the initial marking if and only if the following two conditions hold: (1) there exists a path in the MSCG starting from the initial node, whose sequence of first entries in the edges is $t_{i_{1}} t_{i_{2}} \ldots t_{i_{k}}$; (2) the time instants $\tau_{1}, \tau_{2}, \ldots, \tau_{k}$ satisfy a series of constraints univocally defined, as explained in [1], by the inequalities $\Theta$ in the nodes along the path and by the third entries of the edges along the same path.

Example 1: Consider the TPN system in Fig. 1. A part of its MSCG built according to Algorithm 1 in [1] is shown in Fig. 2.

The time-transition sequence $\left(t_{1}, 1\right)\left(t_{3}, 1.5\right)$ is firable at the initial marking and leads to marking $p_{1}+p_{2}$ where $t_{1}$ and $t_{2}$ are enabled and have a firing delay satisfying the constraints $0 \leq \theta_{1} \leq 2$ and $0 \leq \theta_{2} \leq 0.5$, respectively. Such an evolution corresponds to the path $C_{0} \stackrel{t_{1}, \varepsilon, \Delta_{1} \in[0,2]}{\longrightarrow} C_{1} \stackrel{t_{3}, a, \Delta_{3} \in\left[0,2-\Delta_{1}\right]}{\longrightarrow} C_{2}$ in the MSCG where $\Delta_{1}=1$ and $\Delta_{3}=0.5$, which imply $\tau_{1}=\Delta_{1}=1$ and $\tau_{2}=\Delta_{1}+\Delta_{2}=1.5$.

On the contrary, the time-transition sequence $\left(t_{1}, 1\right)\left(t_{3}, 2.5\right)$ is not firable. This can be easily verified in the

MSCG. Indeed, it logically corresponds to the path $C_{0} \stackrel{t_{1}, \varepsilon, \Delta_{1} \in[0,2]}{\longrightarrow} C_{1} \stackrel{t_{3}, a, \Delta_{3} \in\left[0,2-\Delta_{1}\right]}{\longrightarrow} C_{2}$. However, only $\Delta_{1}=1$ satisfies the timing constraints, while $\Delta_{3}=1.5$ is not an admissible value since, according to the constraint in the second edge of the path, it should be $\Delta_{3} \in\left[0,2-\Delta_{1}\right]=[0,1]$.

\section{SOME REMARKS ON Algorithm 1 IN [1]}

In this section we describe in detail the three issues mentioned in the introduction, which affect Algorithm 1 in [1].

In the following, in accordance with the notation in [1], the generic node $C_{e}$ is labeled with $\left(M_{e}, \Theta_{e}\right)$ where $M_{e}$ is the marking that characterizes it and $\Theta_{e}$ is the set of constraints that characterize the admissible values of the timing delays $\theta_{i}$, for all transitions $t_{i}$ enabled at $M_{e}$. Finally, we denote as $\Delta^{(e)}$ the set of symbolic variables $\Delta$ 's involved in $\Theta_{e}$. 


\section{A. The resulting MSCG may be infinite}

Proposition 5 in [1] claims that, under appropriate assumptions, the Modified State Class Tree (MSCT) built according to Algorithm 1 in [1] (and consequently the MSCG) is finite. This may not be correct in some cases, as shown by the following simple counterexample.

Example 2: Consider again the TPN system in Fig. 1. The repetitive sequence $t_{1} t_{3}$ may fire arbitrarily often in zero time, while keeping unaltered the marking of place $p_{2}$. This leads to an infinite number of classes as shown in Fig. 2. This can be explained in detail as follows. Whenever a new firing of either $t_{1}$ or $t_{3}$ occurs, a new constraint is defined involving a new $\Delta$ variable which takes into account the time delay in which the firing could have occurred with respect to the previous transition firing. This leads to the definition of a new class in the graph. Since each $\Delta$ variable may take a zero value, constraints involving an ever increasing number of different variables $\Delta$ (and consequently also new classes) can be defined. As a result, the MSCG has an infinite number of classes.

\section{B. A problem in the notation}

In [1] the generic symbolic variable $\Delta_{i}$ is denoted as a function of the transition $t_{i}$ appearing in the edge (see e.g., Fig. 2). This may lead to an improper formalisation when transition $t_{i}$ is labeling two or more edges along a path and produces multiple occurences of the same variable $\Delta_{i}$ in a constraint.

This appears evident looking again at the MSCG in Fig. 2. Here variable $\Delta_{1}$ appears twice in the constraint

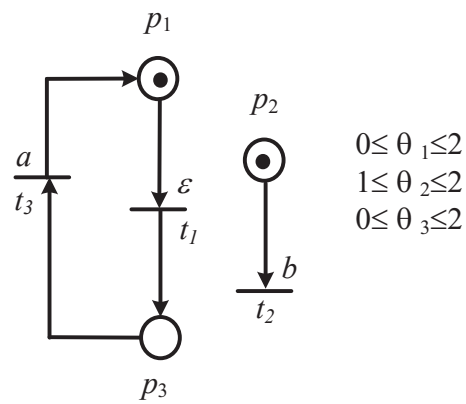

Fig. 1. The TPN system considered in Example 1.

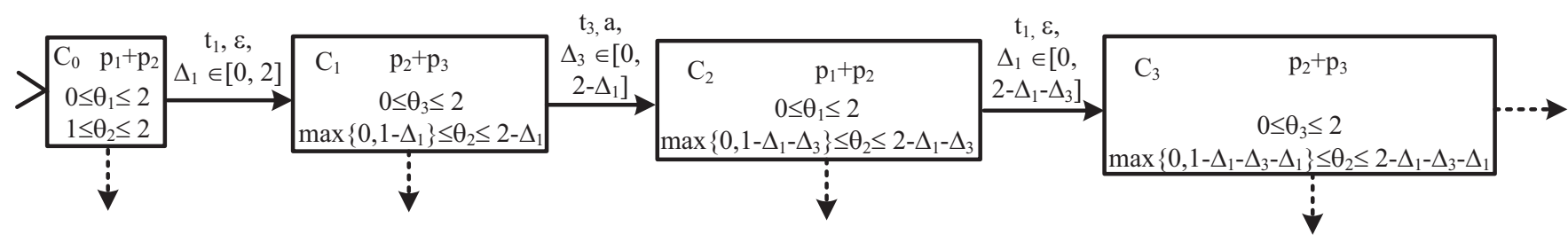

Fig. 2. A part of the MSCG associated with the TPN system in Fig. 1 constructed according to Algorithm 1 in [1]. 
on $\theta_{2}$ in class $C_{3}: \max \left\{0,1-\Delta_{1}-\Delta_{3}-\Delta_{1}\right\} \leq \theta_{2} \leq 2-\Delta_{1}-\Delta_{3}-\Delta_{1}$. Apparently such a constraint could be rewritten as $\max \left\{0,1-2 \Delta_{1}-\Delta_{3}\right\} \leq \theta_{2} \leq 2-2 \Delta_{1}-\Delta_{3}$, which is not correct. Indeed, the same symbol $\Delta_{1}$ denotes two different symbolic variables. One of these variables (let us call it $\Delta_{1}^{\prime}$ ) is subject to the constraint $\Delta_{1}^{\prime} \in[0,2]$ labeling the edge from $C_{0}$ to $C_{1}$. The second variable (let us call it $\Delta_{1}^{\prime \prime}$ ) is subject to the constraint $\Delta_{1}^{\prime \prime} \in\left[0,2-\Delta_{1}^{\prime}-\Delta_{3}\right]$ labeling the edge from $C_{2}$ to $C_{3}$.

\section{Some possible evolutions could be missing}

The last issue concerns the representation of all possible evolutions of the net system. When a node is explored, while constructing the MSCG, it may happen that a logically enabled transition cannot fire because its occurrence will be necessarily preempted by the firing of another enabled transition. In fact, in each node $C_{e}$ the admissible firing delays of enabled transitions satisfy a linear constraint set that depends on the path from the root node to node $C_{e}$. If for all paths leading to $C_{e}$ the lowest admissible value for the firing delay of a logically enabled transition $t$ is greater than the highest admissible value of another enabled transition, then $t$ will never be able to fire from $C_{e}$. In this case we say that transition $t$ is preempted at node $C_{e}$. This leads to the key definition of deficient node and deficient transition at a certain node.

Definition 1: A node $C_{e}$ is deficient if there exists at least one transition that is logically enabled at $M_{e}$, but whose firing will be necessarily preempted by another transition.

Transitions that make node $C_{e}$ deficient are said deficient at node $C_{e}$.

According to Algorithm 1 in [1] each node is explored only once, when it is tagged "new". Therefore, if a transition is deficient when the node is explored, it always remains deficient at that node and no output arc corresponding to it will be created. This may lead to neglect some possible evolutions of the system. Indeed, when new "duplicate" nodes are created ${ }^{1}$, they could generate new paths leading to nodes that have already been explored. If this happens at a node that is deficient, such a node should be explored again to take the new paths into account and determine if some transitions that were previously classified as deficient at such a node, could now be enabled.

Example 3: Consider the TPN system in Fig. 3. The MSCG, constructed according to the Revised Algorithm 1 illustrated in detail in the following Section V, is reported in Fig. 4.f, while the main steps of the construction of the MSCT are shown in Figs. 4.a-e.

Let us focus on node $C_{1}$. It is added when examining node $C_{0}$ (Fig. 4.a). When first analyzed, node $C_{1}$ is tagged "deficient" since only one $\left(t_{4}\right)$ of the two transitions logically enabled at marking $p_{2}+p_{4}\left(t_{4}\right.$ and $\left.t_{5}\right)$, may actually fire (Fig. 4.b). In particular, $t_{5}$ cannot fire since the smallest admissible value of its lower bound is equal to 2 (it corresponds to $\Delta_{0}=1$ ) and is thus greater than the upper bound of $t_{4}$, which is equal to 1 .

Then, when node $C_{2}$ is examined, node $C_{1}^{\prime}$, which is a duplicate of $C_{1}$, is added to the tree (Fig. 4.c). Node $C_{1}^{\prime}$ will be merged with $C_{1}$ when constructing the graph (Fig. 4.f), thus the path leading to it also leads to node

\footnotetext{
${ }^{1}$ The tag "duplicate" is used here to avoid exploring nodes already considered. Duplicate nodes are then merged when constructing the graph starting from the tree.
} 
$C_{1}$ in the graph. Therefore, differently from what is done in [1], $C_{1}$ should be examined again to see if some of the deficient transitions ( $t_{5}$ in this case) cease being deficient thanks to the new path. In the case at hand, this is actually the case and node $C_{1}$ ceases being deficient as highlighted in Fig. 4.e. Indeed, according to the new path that leads to $C_{1}^{\prime}$ (and thus to $C_{1}$ in the graph) the smallest admissible value of the lower bound of the delay of $t_{5}$ is equal to 1 (which corresponds to ${ }^{2} \Delta_{2}=2$ ) and is thus equal to the upper bound of $t_{4}$, which is also equal to 1 .

We finally notice that nodes $C_{0}$ and $C_{2}$ are tagged "deficient" when first examined, and remain deficient for ever. In particular, no further path leading to them is created while constructing the MSCT, so they are not examined further.

\section{REVISED NOTATION, EQUIVALENT NODES, AND ISOMORPHISM RELATIONSHIP}

In this section we first provide a solution to the problem in the notation discussed in the previous Subsection III-B. Then, we formalize the notion of equivalent nodes (provided only in a discursive way in [1]) and clarify the requirement of including additional information on some edges of the MSCG.

An easy solution to the problem in the notation consists in defining symbolic variables $\Delta$ 's on the edges exiting from a certain node, as a function of the node itself. In this new formalism, given a node $C_{e}$ all variables $\Delta$ 's associated with output edges from $C_{e}$ are denoted as $\Delta_{e}$, regardless of the transition with which they are associated. This is also consistent with the physical meaning of such variables: $\Delta_{e}$ represents the time spent by the net system in class $C_{e}$. Clearly, depending on the transition associated with the edge, the constraints to which they are subject, are typically different.

This revision in the notation requires a modification in the algorithm to construct the MSCT. To explain this (and the proposed solution) we preliminarily provide a formal definition of the notion of equivalent classes.

Definition 2: Given a node $C_{e}$ labeled with $\left(M_{e}, \Theta_{e}\right)$ and a node $C_{q}$ labeled with $\left(M_{q}, \Theta_{q}\right), C_{e}$ and $C_{q}$ are said to be equivalent if $M_{e}=M_{q}$, and the set of constraints $\Theta_{e}$ and $\Theta_{q}$ are isomorphic, i.e., there exists a bijective function that defines a one to one mapping between the symbolic variables $\Delta$ 's in $\Delta^{(e)}$ and the

${ }^{2}$ Looking at the edge from $C_{2}$ to $C_{1}^{\prime}$, we read that $\Delta_{2} \in[0.2]$. In the same edge we find out (as the fourth label) an isomorphism relationship, namely $\Delta_{0}:=\Delta_{2}$. As explained in detail in the following, this means that in all the constraints in the input node of the edge, namely $C_{1}^{\prime}, \Delta_{0}$ should be replaced by $\Delta_{2}$.

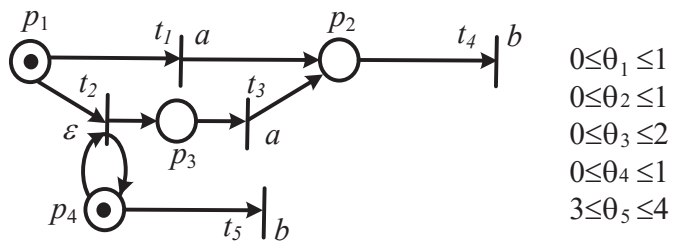

Fig. 3. The TPN system considered in Example 3. 

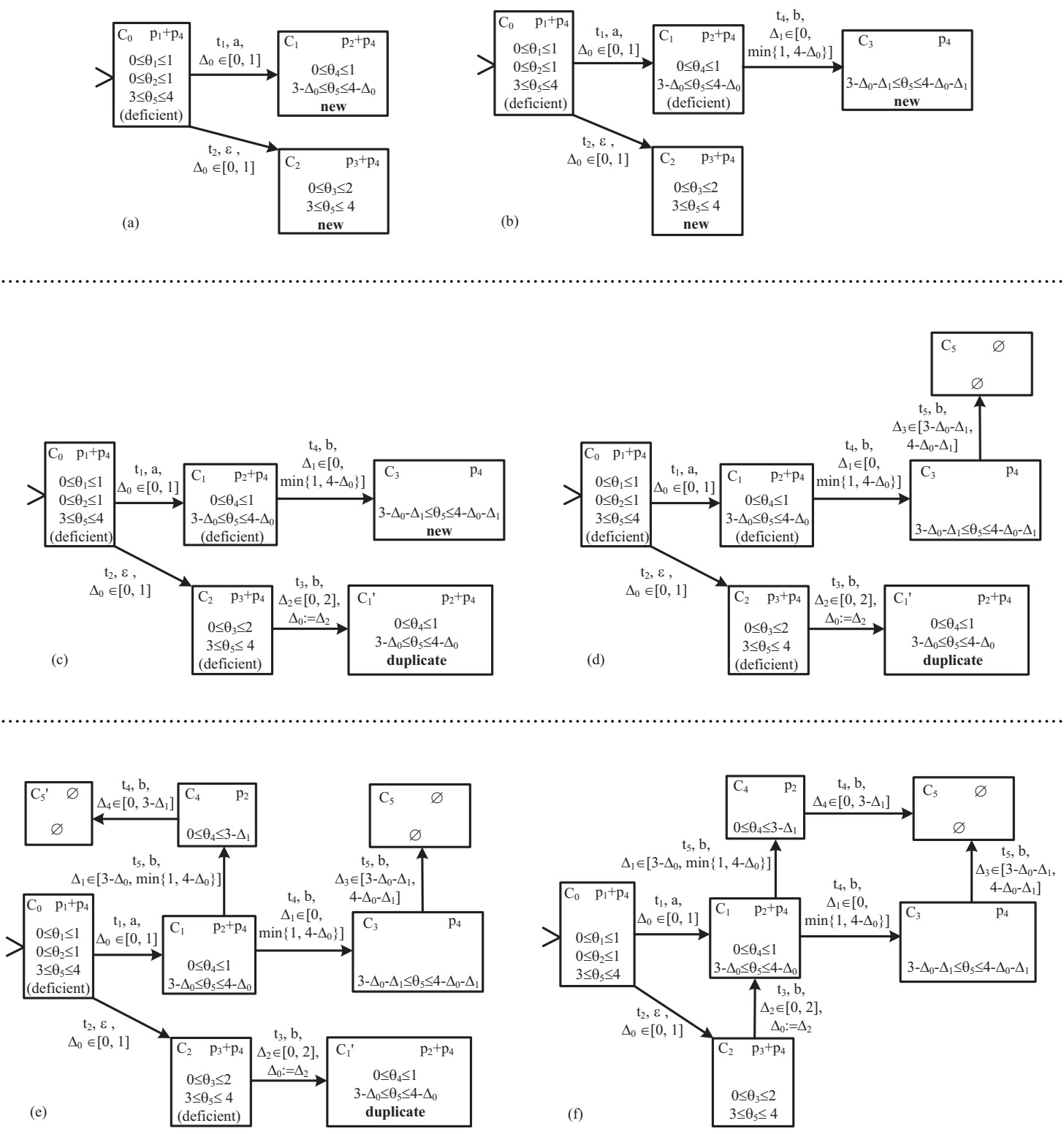

Fig. 4. The MSCG (Fig. (f)) of the TPN system in Fig. 3, built according to Revised Algorithm 1, and the most significant intermediate steps in its construction (Figs. (a) to (e)).

symbolic variables $\Delta^{\prime}$ s in $\Delta^{(q)}$, so that the set of constraints $\Theta_{e}$ is converted into the set of constraints $\Theta_{q}$, and viceversa.

In the following we denote by $f_{e q}: \Delta^{(e)} \rightarrow \Delta^{(q)}$ the one to one mapping between the variables in the set $\Delta^{(e)}$ and the variables in the set $\Delta^{(q)}$.

When constructing the MSCT, several nodes belonging to the same class of the equivalence relation previously defined may be encountered. The first of this node, say $C_{e}$, is considered as representative of the class and the 
possible evolutions from it will be further explored. All other nodes are labeled "duplicate" and will not be further explored: thus they will be leaves of the MSCT. In addition, to keep track of the isomorphism between a duplicate node $C_{q}$ and its representative node $C_{e}$, we add to the label of the edge entering $C_{q}$ a fourth entry $\Delta^{(e)}:=f_{e q}\left(\Delta^{(q)}\right)$.

Example 4: Consider again the TPN system in Fig. 3. As already discussed in Example 3, Fig. 4 illustrates the main steps in the construction of the MSCT using Revised Algorithm 1, as well as the resulting MSCG.

Let us focus on node $C_{1}$. It is added during the first iteration of Algorithm 1, when examining node $C_{0}$ (Fig. 4.a). Then, when exploring node $C_{2}$, a node equivalent to $C_{1}$, namely $C_{1}^{\prime}$, is computed. Therefore, $C_{1}^{\prime}$ is tagged "duplicate" (Fig. 4.c) and the isomorphism relationship $\Delta_{0}:=\Delta_{2}$ is added as the fourth entry of the edge from $C_{2}$ to $C_{1}^{\prime}$.

Node $C_{1}$ is then merged with node $C_{1}^{\prime}$ when constructing the MSCG strarting from the MSCT (Fig. 4.f) and the isomorphism relationship mentioned above (appearing in the edge from $C_{2}$ to $C_{1}$ in the MSCG) specifies how to write the constraints involving the timing delays of transitions $t_{4}$ and $t_{5}$ when following the path $C_{0} \stackrel{t_{2}, \varepsilon, \Delta_{0} \in[0,1]}{\longrightarrow} C_{2} \stackrel{t_{3}, b, \Delta_{2} \in[0,2]}{\longrightarrow} C_{1}$ in the MSCG. In particular, it specifies that all constraints written in terms of $\Delta_{0}$ in node $C_{1}$ and in its output edges, should be rewritten replacing $\Delta_{0}$ with $\Delta_{2}$ when $C_{1}$ is reached via $C_{2}$.

\section{THE REVISED ALGORITHM FOR THE CONSTRUCTION OF THE MSCT}

In this section we provide a revised version of Algorithm 1 in [1] and prove that, under appropriate assumptions, the resulting MSCG is finite. For the sake of clarity and conciseness, the proposed algorithm uses a function, named "Look for new nodes", which is called twice with different input arguments. In more detail, Revised Algorithm 1 summarizes the main steps of the new algorithm for the construction of the MSCT, while Algorithm 2 summarizes the main steps of the function "Look for new nodes". Such a function has two input arguments: a node $C_{k}$ and a set of duplicate nodes Duplicate. Its role is that of looking for new nodes in the tree starting from the exploration of node $C_{k}$. Furthermore, as discussed in more detail in the rest of the section, the second argument takes different values depending on the fact that $C_{k}$ is explored for the first time, or it has been already explored.

Note that in Revised Algorithm 1 and in Algorithm 2, $\mathcal{A}(M)$ denotes the set of transitions logically enabled at $M$.

\section{A. Explanation of Algorithm 1}

Algorithm 1 starts by defining as root node of the tree the class $C_{0}$, which is initially tagged "new".

Then two sets are introduced and initialized at the empty set (Steps 2 and 3, respectively):

- Def is the current set of deficient nodes;

- Dup is the current set of duplicate nodes.

The above two sets are then updated while executing Algorithm 2. 


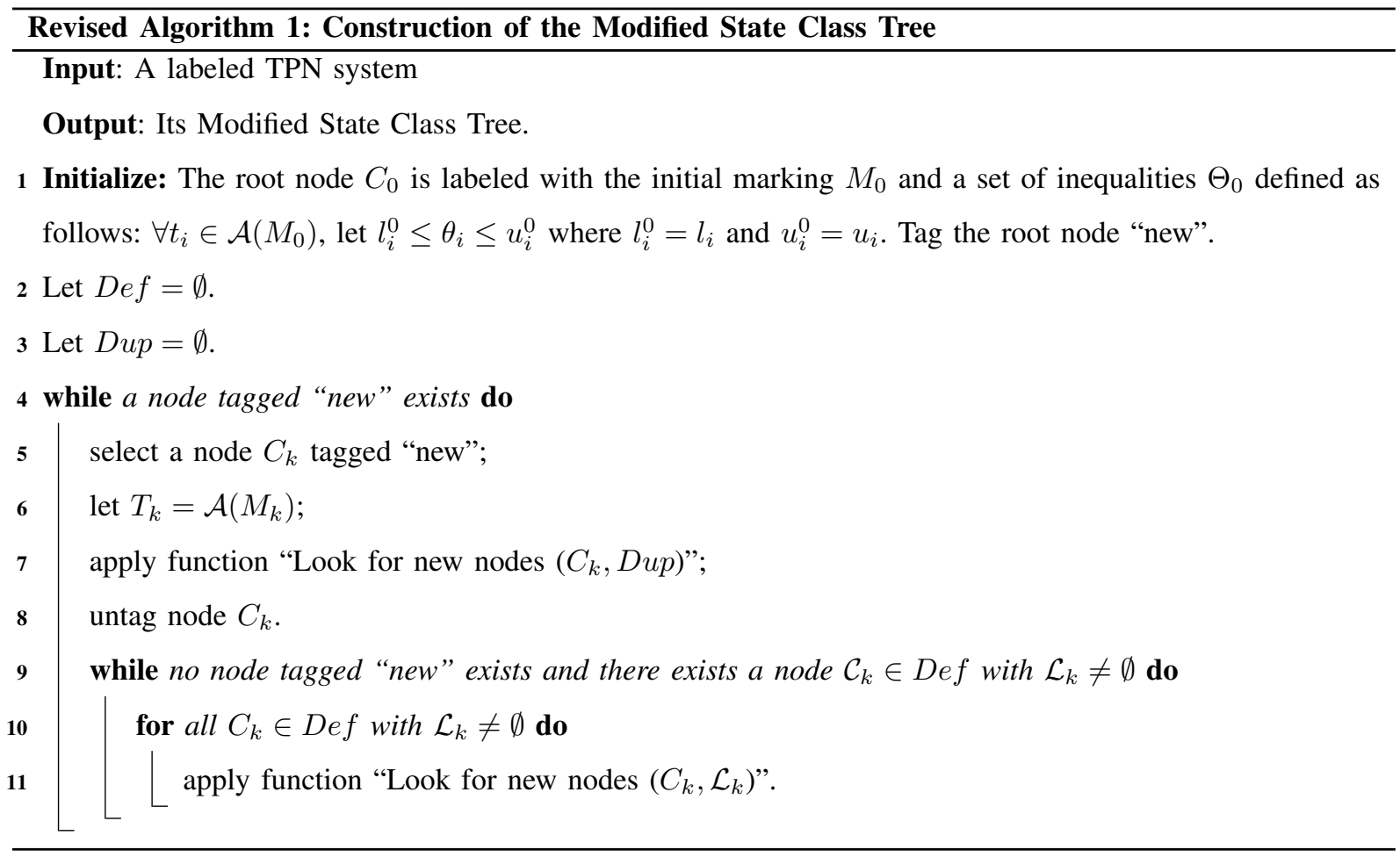

While nodes tagged "new" exist, one of such nodes is selected. Let's call it $C_{k}$ (Step 5). A set of transitions $T_{k}$ is associated with node $C_{k}$ and it is initialized at $T_{k}=\mathcal{A}\left(M_{k}\right)$ (Step 6).

- Set $T_{k}$ contains the deficient transitions at node $C_{k}$. More precisely, the first time node $C_{k}$ is selected at Step 5 of Algorithm 1, namely when $C_{k}$ is tagged "new”, $T_{k}$ is initialized at $\mathcal{A}\left(M_{k}\right)$ (Step 6 of Algorithm 1). Then, whenever Algorithm 2 (Step 3) finds out that a transition in $T_{k}$ is not deficient at node $C_{k}$, such a transition is removed from set $T_{k}$ (Step 4 of Algorithm 2). Therefore, once $C_{k}$ has been explored, $T_{k}$ contains the set of transitions that are currently deficient at node $C_{k}$. As a result, $C_{k}$ is classified deficient at a certain iteration if and only if at that iteration it is $T_{k} \neq \emptyset$.

At Step 7, Algorithm 2 is executed having as input arguments $C_{k}$ and Dup. In simple words, new nodes are eventually created in the tree exploring node $C_{k}$ and taking into account the whole current set of duplicate nodes. After that, node $C_{k}$ is untagged (Step 8).

The second while loop is based on the definition of set $\mathcal{L}_{k}$ which has the following physical meaning:

- $\mathcal{L}_{k}$ is associated with the generic deficient node $C_{k}$ and contains the duplicate nodes that have been created in the tree after node $C_{k}$ has been explored the last time.

The second while loop applies when no node tagged "new" exists, but there exists at least one deficient node $C_{k}$ whose set $\mathcal{L}_{k}$ is not empty: this means that $C_{k}$ is not new, but, since it is deficient and some duplicate nodes have been added to the tree after it has been examined the last time, it could happen that some transitions that are currently deficient at node $C_{k}$, cease being deficient thanks to the new paths that such duplicate nodes have 


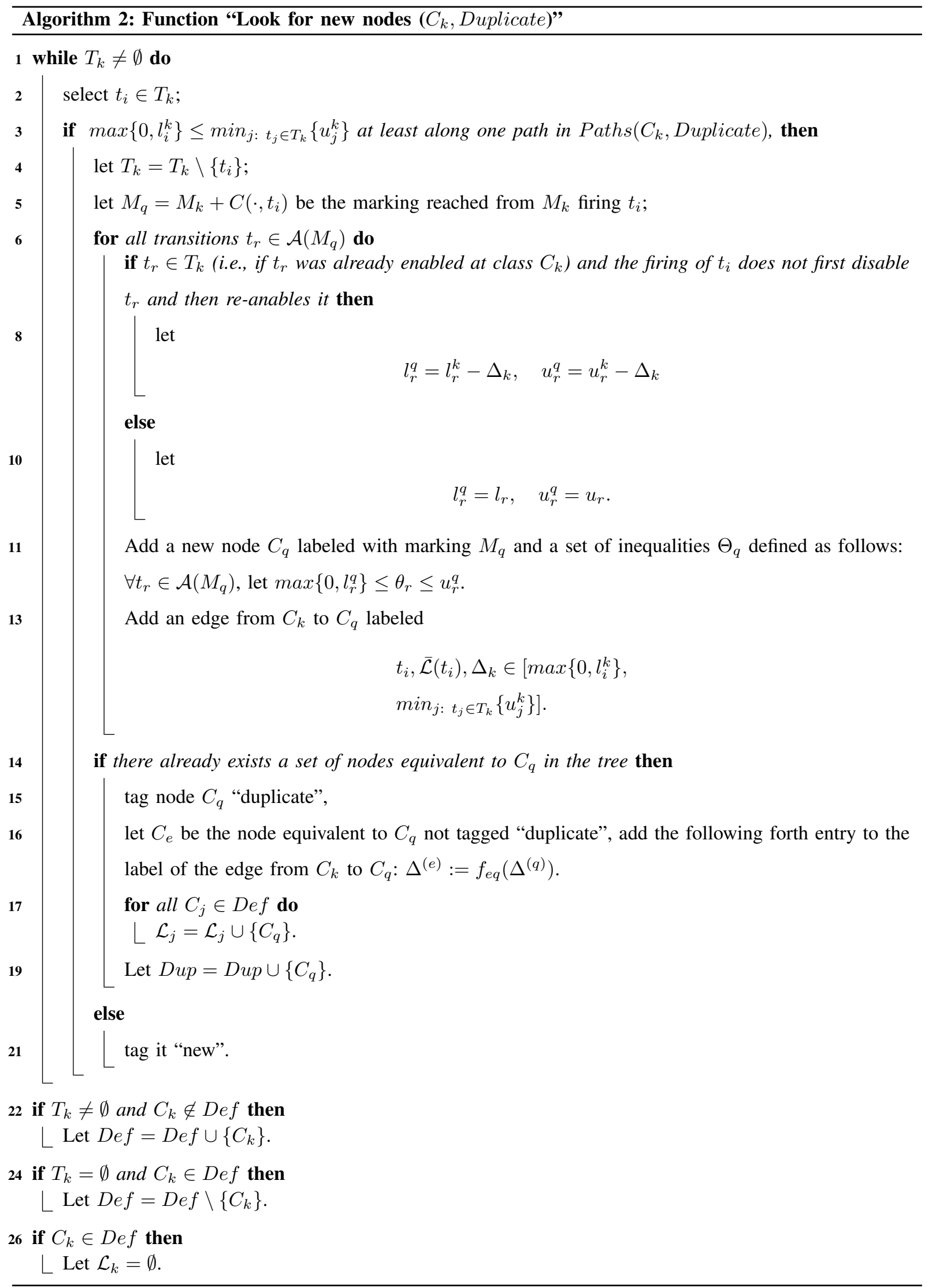


generated. The role of this while loop is that of investigating if this actually occurs and, if such is the case for some node $C_{k}$, apply function "Look for new nodes" with input arguments node $C_{k}$ and its corresponding set $\mathcal{L}_{k}$.

\section{B. Explanation of Algorithm 2}

Algorithm 2, as the name of the function highlights, looks for new nodes in the tree. This is done exploring a generic node $C_{k}$. If this happens when node $C_{k}$ is tagged "new", namely it has never been explored before, the second argument of the function Duplicate is equal to Dup, i.e., all duplicate nodes should be considered. On the contrary, if $C_{k}$ is not tagged "new", namely it has been already explored, Duplicate is equal to $\mathcal{L}_{k}$, i.e., only the duplicate nodes that have been created after $C_{k}$ has been examined the last time, should be considered.

The effect of Algorithm 2 is not only that of eventually creating new nodes and edges. It also updates: sets $T_{k}$ and $\mathcal{L}_{k}$ associated with the current node $C_{k}$, sets $\mathcal{L}_{j}$ associated with the current deficient nodes $C_{j} \in D e f$, sets Dup and Def.

Algorithm 2 uses the function Paths $\left(C_{k}\right.$,Duplicate $)$ defined as follows:

- Paths $\left(C_{k}\right.$, Duplicate $)$ is a function that returns all paths in the current MSCG obtained merging duplicate nodes in the set Duplicate, and terminating in node $C_{k}$. In particular, such paths are defined moving backward towards $C_{0}$, untill all variables $\Delta$ 's defining the set of admissible firing delays of the transitions enabled at $C_{k}$ have been encountered, taking into account isomorphism relationships, if any.

We point out that Paths $\left(C_{k}\right.$, Duplicate) always returns a finite number of paths. In fact, even if a cycle originates while merging duplicate nodes, when moving backward along the cycle, before the cycle is completed, the involved timers are reset and no trace of the upstream variables $\Delta$ 's remains. Indeed, if by absurd, one timer is not reset, while moving backward, the number of variables $\Delta$ 's associated with it would grow indefinitely. However, this is in contrast with the fact that a cycle is defined via a duplicate node and an isomorphism relationship associated with the input edge closing the cycle.

Let us now explain Algorithm 2 step by step.

It examines the set of transitions in $T_{k}$ (Step 1). More precisely, given a transition $t_{i} \in T_{k}$, it checks if there exists at least one path in Paths $\left(C_{k}\right.$, Duplicate) that enables it (Step 3). If such is the case, $t_{i}$ is removed from $T_{k}$ (Step 4) and the marking $M_{q}$ reached firing $t_{i}$ at $M_{k}$ is computed (Step 5). Lower and upper bounds of all transitions logically enabled at marking $M_{q}$ are updated at Step 8 or 10.

At Step 11 a new node $C_{q}$ labeled with marking $M_{q}$ and set of inequalities $\Theta_{q}$ is added. Step 13 defines the label of the edge from $C_{k}$ to $C_{q}$, to whom an additional entry may be eventually added in Step 16.

Step 16 clarifies how to handle the case in which $C_{q}$ is a duplicate node. In particular, it defines the isomorphism relationship to be added as a fourth entry on the edge from $C_{k}$ to $C_{q}$ in accordance with the discussion in the previous section.

At Step 17 all sets $\mathcal{L}_{j}$ associated with the current set of deficient nodes (nodes in Def) are updated, including in all of them the new node $C_{q}$. Analogously, $C_{q}$ is included in the set Dup at Step 19. 
If the new node $C_{q}$ is not duplicate, it is tagged "new" at Step 21.

Finally, if the set $T_{k}$ is not empty after all transitions in it have been examined and $C_{k}$ was not already in $D e f$, then $C_{k}$ is added to Def. Note that this could only occur when node $C_{k}$ is examined for the first time. A node initially assigned to $D e f$, may later be removed if its set $T_{k}$ becomes empty (Step 24).

The algorithm terminates at Step 26 which imposes that set $\mathcal{L}_{k}$ is reset to the empty set if $C_{k}$ is still a deficient node: all duplicate nodes computed up to now have been already considered when examining $C_{k}$.

Example 5: Consider again the TPN system in Fig. 3. The main steps in the construction of the MSCT, built according to Revised Algorithm 1, and the resulting MSCG are reported in Fig. 4 as already mentioned in the previous examples.

- At Step 1 of Revised Algorithm 1, node $C_{0}$ is created and tagged "new": it contains the initial marking $M_{0}$ and the timing constraints of the transitions logically enabled at $M_{0}$ are those defined by function $Q(\cdot)$. Sets Def and Dup are initialized at the empty set (Steps 2 and 3).

- Node $C_{0}$ is examined since it is the only node of the tree and it is tagged "new" (Steps 4 and 5). Set $T_{0}$ is initialized at the set of transitions logically enabled at $p_{1}+p_{4}$, namely, it is $T_{0}=\left\{t_{1}, t_{2}, t_{5}\right\}$. Only transitions $t_{1}$ and $t_{2}$ satisfy the if condition at Step 3 of Algorithm 2. In particular, their firing leads to nodes $C_{1}$ and $C_{2}$, respectively, which are added to the tree and tagged "new" as shown in Fig. 4.a. Set $T_{0}$ becomes equal to $T_{0}=\left\{t_{5}\right\}$, therefore at Step 22 of Algorithm 2 set Def is updated at Def $=\left\{C_{0}\right\}$. At Step 26 of the same algorithm a new set $\mathcal{L}_{0}$ is introduced and initialized at the empty set. Finally, node $C_{0}$ is untagged at Step 8 of Revised Algorithm 1.

- Assume that node $C_{1}$ is selected at Step 5 of Revised Algorithm 1. Set $T_{1}$ is initialized at $T_{1}=\left\{t_{4}, t_{5}\right\}$. However, only $t_{4}$ satisfies the if condition in Step 3 of Algorithm 2. Therefore $T_{1}$ is updated at $T_{1}=\left\{t_{5}\right\}$ and a new node, denoted $C_{3}$, is added to the tree and tagged "new" as shown in Fig. 4.b. At Step 22 of Algorithm 2 , the set of deficient nodes $D e f$ is updated at $D e f=\left\{C_{0}, C_{1}\right\}$. Furthermore, at Step 26 of the same algorithm, a new set $\mathcal{L}_{1}$ is introduced and initialized at the empty set.

- Assume that node $C_{2}$ is selected at Step 5 of Revised Algorithm 1. Set $T_{2}$ is initialized at $T_{2}=\left\{t_{3}, t_{5}\right\}$. However, only $t_{3}$ satisfies the if condition in Step 3 of Algorithm 2. Therefore $T_{2}$ becomes equal to $\left\{t_{5}\right\}$ and a new node is added to the tree. As shown in Fig. 4.c such a node is denoted as $C_{1}^{\prime}$ to point out that it is equivalent to node $C_{1}$. Such a node is tagged "duplicate". Furthermore, in accordance with Step 16 of Algorithm 2, the isomorphism relationship $\Delta_{0}:=\Delta_{2}$ is added as the fourth enrty of the edge from $C_{2}$ to $C_{1}^{\prime}$. A new set $\mathcal{L}_{1}^{\prime}$ is introduced and initialized at the current set Dup, namely, it is $\mathcal{L}_{1}^{\prime}=$ Dup $=\emptyset$. At Step 17, sets $\mathcal{L}_{0}$ and $\mathcal{L}_{1}$ are updated, including the duplicate node $C_{1}^{\prime}$, namely it becomes $\mathcal{L}_{0}=\mathcal{L}_{1}=\left\{C_{1}^{\prime}\right\}$. Set Dup is updated at Step 19. In particular, it is Dup $=\left\{C_{1}^{\prime}\right\}$. At Step 22, set Def is updated at Def $=\left\{C_{0}, C_{1}, C_{2}\right\}$. A new set $\mathcal{L}_{2}$ is introduced and initialized at the empty set at Step 26. Finally, node $C_{2}$ is untagged at Step 8 of Revised Algorithm 1.

- At this point, there exists only one node tagged "new", namely $C_{3}$ therefore it is selected at Step 5 of Revised Algorithm 1. Set $T_{3}$ is initialized at $\left\{t_{5}\right\}$ since $t_{5}$ is the only transition enabled at $p_{4}$. Transition $t_{5}$ 
satisfies the if condition in Step 3 of Algorithm 2. Therefore $T_{3}$ becomes equal to the empty set. A new node is computed, denoted as $C_{5}$, which corresponds to the empty marking. Steps 22 to 26 produce no effect since none of the if condition is satisfied. Therefore sets Def and Dup remain unaltered and node $C_{3}$ is untagged.

- Again, there exists only one node tagged "new", namely $C_{5}$ therefore it is selected at Step 5 of Revised Algorithm 1. However, since it enables no transition $\left(T_{5}=\emptyset\right)$, the effect of Algorithm 2 is simply that of removing the tag "new" from it as shown in Fig. 4.d.

- Now, no node tagged "new" exist. However, Def $=\left\{C_{0}, C_{1}, C_{2}\right\}$ and $\mathcal{L}_{0}=\mathcal{L}_{1}=\left\{C_{1}^{\prime}\right\}$ (while $\mathcal{L}_{2}=\emptyset$ ), therefore the condition on the while loop at Step 9 of Revised Algorithm 1 is satisfied. In more detail, nodes $C_{0}$ and $C_{1}$ also satisfy the condition on the for loop in Step 10.

Assume that node $C_{0}$ is considered first. Since function $\operatorname{Path}\left(C_{0}, \mathcal{L}_{0}\right)=\operatorname{Path}\left(C_{0}, \mathcal{C}_{1}^{\prime}\right)$ returns no path (duplicate node $\mathcal{C}_{1}^{\prime}$ created no new path going from $\mathcal{C}_{0}$ to $\mathcal{C}_{0}$ ), no new node is added. The only effect of Algorithm 2 is that of updating $\mathcal{L}_{0}$ at the empty set.

- Let us now consider node $C_{1}$. Function $\operatorname{Path}\left(C_{1}, \mathcal{L}_{1}\right)=\operatorname{Path}\left(C_{1}, \mathcal{C}_{1}^{\prime}\right)$ returns the path $C_{0} C_{2} C_{1}$. Furthermore, transition $t_{5} \in T_{1}$ satisfies the if condition of Step 3 of Algorithm 2. Therefore $T_{1}$ becomes equal to the empty set. A new node, denoted as $C_{4}$ in Fig. 4.e, is computed and tagged "new". Node $C_{1}$ is removed from set Def, which becomes equal to Def $=\left\{C_{0}, C_{2}\right\}$ at Step 24 of Algorithm 2.

- Now, there exists again a node tagged "new" $\left(C_{4}\right)$ so Revised Algorithm 1 executes the first while loop. At node $C_{4}$ only one transition is enabled $\left(t_{4}\right)$ and it leads to node $C_{5}^{\prime}$, which is a duplicate of $C_{5}$.

At this point no node tagged "new" exist. Furthermore, Def $=\left\{C_{0}, C_{2}\right\}$ but $\mathcal{L}_{0}$ and $\mathcal{L}_{2}$ are both equal to the empty set so the conditions to enter in both while loops are violated. As a result Revised Algorithm 1 stops and the tree is complete.

\section{Finiteness of the MSCG built using Revised Algorithm 1}

We conclude this section proving that Revised Algorithm 1 terminates in a finite number of steps, which in turns implies that the resulting MSCG is finite, provided that the following two assumptions hold: Assumption A1 in [1] (the TPN is bounded), plus an additional assumption that prevents situations like the one pointed out in Subsection III-A. In particular, if we denote as

$$
T_{0}=\left\{t_{i} \in T: l_{i}=0\right\},
$$

the additional assumption imposes that:

(B) no sequence $\sigma \in T_{0}^{*}$ is repetitive.

By Assumption B, for a given bounded net the maximum number of consecutive firings of transitions in $T_{0}$, denoted as $r_{0}$, is finite. Clearly, $r_{0}$ is a function of the initial marking.

In simple words, the MSCG is finite if there exist no repetitive sequence may fire in zero time. We notice that such an assumption does not provide a limitation in practical applications because this is just a necessary and sufficient condition to rule out the possibility that the model is zeno and consequently not suitable to describe 
a real physical system. Indeed, events represent operations on the system that require a non null time to be executed. As an example, in a manufacturing system, events may be the processing of a part by a machine, or the failure of a certain operation, or some activities performed by a human operator, and so on.

We now provide a lemma which is fundamental to demonstrate the finiteness of the MSCG. It claims that no transition may remain enabled, without firing, along a path of the MSCG of arbitrary lentgh.

Lemma 1: Consider a bounded TPN $\left\langle N_{d}, M_{0}\right\rangle$ and its MSCG built according to Revised Algorithm 1. Under Assumption $\mathrm{B}$, no transition $t \in T$ remains enabled without firing along a path of length greater than or equal to

$$
\mu=\left(r_{0}\left|T \backslash T_{0}\right|+1\right)\left\lceil\frac{u_{\max }}{l_{\min }}\right\rceil
$$

where

$$
l_{\min }=\min _{t_{i} \in T \backslash T_{0}} l_{i}, \quad u_{\max }=\max _{t_{i} \in T \backslash T_{0}} u_{i} .
$$

Proof: We prove this by contradiction. Assume that there exists a transition $\bar{t}$ which remains enabled without firing, along an arbitrarily long path in the MSCG. Let

$$
C_{i_{0}} \stackrel{t_{j_{0}}, \mathcal{L}\left(t_{j_{0}}\right), \Delta_{i_{0}} \in[\cdots]}{\longrightarrow} C_{i_{1}} \stackrel{t_{j_{1}}, \mathcal{L}\left(t_{j_{1}}\right), \Delta_{i_{1}} \in[\cdots]}{\longrightarrow} C_{i_{2}} \rightarrow \ldots
$$

be such a path, where the first node of the path, $C_{i_{0}}$, corresponds to the node where transition $\bar{t}$ is newly enabled. Since $\bar{t}$ remains enabled along the above path, a constraint on its firing delay $\bar{\theta}$ appears in all nodes of the path. In particular, if we denote by $\bar{l}$ and $\bar{u}$ the static bounds of $\bar{t}$, the constraint on $\bar{\theta}$ in the generic node $C_{r}$ of the path, is equal to

$$
\max \left\{0, \bar{l}-\sum_{q=0}^{r-1} \Delta_{i_{q}}\right\} \leq \bar{\theta} \leq \bar{u}-\sum_{q=0}^{r-1} \Delta_{i_{q}} .
$$

Now, by Assumption B, the number of consecutive firings of transitions with a null firing delay in the path at hand, is bounded and can be easily computed. Indeed, the longest sequence of transitions that fire consecutively in zero time and may appear in the path, have the following form:

$$
\sigma_{0,1} t_{\varrho_{1}} \sigma_{0,2} t_{\varrho_{2}} \ldots t_{\varrho_{k}} \sigma_{0, k}
$$

where $\sigma_{0, i} \in T_{0}^{*}, t_{\varrho_{i}} \in T \backslash T_{0} \backslash\{\bar{t}\}$, for $i=1, \ldots, k$, and $t_{\varrho_{i}} \neq t_{\varrho_{j}}$ for all $i \neq j$. The last condition follows from the fact that, when a transition fires, its timer is reset and, in the case of a transition in $T \backslash T_{0}$, the next firing may only occur after a time interval which is greater than zero by definition. As a result, the integer $k$ in (4) satisfies

$$
k=\left|T \backslash T_{0}\right|-1
$$

where -1 originates from the fact that $\bar{t}$ is not firing by assumption. Therefore, the max length of a sequence defined as in Eq. (4), is equal to $r_{0}\left(\left|T \backslash T_{0}\right|-1\right)+r_{0}=r_{0}\left|T \backslash T_{0}\right|$.

After the firing of a sequence defined as in Eq. (4), a transition in $T \backslash T_{0} \backslash\{\bar{t}\}$ fires after a delay greater than or equal to $l_{\min }$. This enables us to conclude that, after a number of firings equal to $r_{0}\left|T \backslash T_{0}\right|+1$, all 
admissible values for the timer of $\bar{t}$ are certainly smaller than $\bar{u}-l_{\text {min }}$. Repeating this reasoning, after at most $\bar{\mu}$ firings, where $\bar{\mu}$ is equal to

$$
\bar{\mu}=\left(r_{0}\left|T \backslash T_{0}\right|+1\right)\left\lceil\frac{\bar{u}}{l_{\min }}\right\rceil,
$$

all admissible values of the timer of $\bar{t}$ is certainly smaller than $l_{\text {min }}$, thus the transition must fire after at most $r_{0}\left|T \backslash T_{0}\right|+1$ additional firings. The statement follows from the fact that $\forall \bar{t} \in T \backslash T_{0}$, it is

$$
\bar{\mu} \leq \mu=\left(r_{0}\left|T \backslash T_{0}\right|+1\right)\left\lceil\frac{u_{\max }}{l_{\min }}\right\rceil .
$$

Proposition 1: Let $\left\langle N_{d}, M_{0}\right\rangle$ be a bounded TPN. Under Assumption B, the MSCT as defined by Revised Algorithm 1 is finite.

Proof: Each node $C_{e}$ of the graph is labeled by pair $\left(M_{e}, \Theta_{e}\right)$ where $M_{e}$ is a reachable marking and $\Theta_{e}$ is a constraint set. The result follows from two facts. First, being the TPN bounded, the number of reachable markings is finite. Secondly, by Lemma 1 in each inequality in $\Theta_{e}$ the number of $\Delta$ variables is at most equal to $\mu$, hence, the number of possible constraint sets is finite.

Clearly, if the MSCT is finite, the MSCG is finite as well, since it is obtained simply merging the duplicate nodes.

We conclude this section mentioning that in [1] the static bounds of all transitions were assumed to be rational numbers. As shown in the above proposition, such a condition is not necessary to guarantee the finiteness of the MSCG.

\section{Vi. CONCLusions}

In this Technical Note we made some remarks on the paper by Basile et al. [1]. In particular, the concerns are related to a graph, called Modified State Class Graph, which should, under certain assumptions, summarize all possible evolutions of a labeled Time Petri net system. Three are the main criticalities in the algorithm in [1]: the resulting MSCG may be infinite, there is a problem in the notation used in [1], some possible evolutions could be missing. A revised version of the algorithm is proposed in this paper and it is proved to be finite under an additional assumption, not mentioned in [1].

We finally observed that the procedures for state estimation and fault diagnosis proposed in [1], as well as the diagnosability analysis approach in [2], which use the Modified State Class Graph as the basis tool, are not affected by the proposed modifications in its definition and in its rules of construction.

\section{ACKNOWLEDGEMENT}

This work was supported by the National Natural Science Foundation of China under Grant Nos. 61803246, 61472295, and 61703321, the Science and Technology Development Fund, MSAR, under Grant No. 078/2015/A3, and the Natural Science Foundation of Shaanxi University of Science and Technology under Grant Nos. 2016BJ15 and $2017 \mathrm{BJ}-39$. 


\section{REFERENCES}

[1] F. Basile, M.P. Cabasino, and C. Seatzu. "State estimation and fault diagnosis of time labeled Petri net systems with unobservable transitions," IEEE Transactions on Automatic Control, vol. 60, no. 4, pp. 997-1009, 2015.

[2] F. Basile, M.P. Cabasino, and C. Seatzu. "Diagnosability analysis of labeled time Petri net systems," IEEE Transactions on Automatic Control, vol. 62, no. 3, pp. 1384-1396, 2017.

[3] T. Murata. "Petri nets: Properties, analysis and applications," in Proc. IEEE, vol. 77, no. 4, pp. 541-580, 1989. 